\title{
Identification of medium-sized genomic deletions with low coverage, mate-paired restricted tags
}

\author{
Qiang Gong ${ }^{1,2}$, Yong Tao ${ }^{1}$, Jian-Rong Yang ${ }^{3}$, Jun Cai ${ }^{1}$, Yunfei Yuan ${ }^{4}$, Jue Ruan ${ }^{1}$, Jin Yang ${ }^{5}$, Hailiang Liu ${ }^{3}$, \\ Wanghua Li $i^{1,2}$, Xuemei Lư ${ }^{1,5}$, Shi-Mei Zhuang ${ }^{3}$, San Ming Wang ${ }^{6}$ and Chung-I Wu ${ }^{1,7^{*}}$
}

\begin{abstract}
Background: Genomic deletions are known to be widespread in many species. Variant sequencing-based approaches for identifying deletions have been developed, but their powers to detect those deletions that affect medium-sized regions are limited when the sequencing coverage is low.

Results: We present a cost-effective method for identifying medium-sized deletions in genomic regions with low genomic coverage. Two mate-paired libraries were separately constructed from human cancerous tissue to generate paired short reads (ditags) from restriction fragments digested with a 4-base restriction enzyme. A total of $3 \mathrm{~Gb}$ of paired reads (1.0x genome size) was collected, and 175 deletions were inferred by identifying the ditags with disorder alignments to the reference genome sequence. Sanger sequencing results confirmed an overall detection accuracy of $95 \%$. Good reproducibility was verified by the deletions that were detected by both libraries.

Conclusions: We provide an approach to accurately identify medium-sized deletions in large genomes with low sequence coverage. It can be applied in studies of comparative genomics and in the identification of germline and somatic variants.
\end{abstract}

Keywords: Medium-sized deletion, Restriction enzymes, Next generation sequencing, Structural variation

\section{Background}

A major objective of genomic studies is to characterize genetic variations. The types of variants include single nucleotide polymorphisms (SNPs), micro-insertions/deletions (indels) and large, structural variations of deletions, insertions, translocations and inversions [1-4].

Traditionally, deletions at the megabase and submegabase levels are characterized by positional cloning and microarray technologies [5-7]. With the rapid progress of next-generation sequencing (NGS) technology $[8,9]$, a strategy of paired-read sequencing has been developed [10-12]. Several methods have been developed to characterize the breakpoints of structural variants, including analysis of the so-called 'split reads' that map to different loci of the reference sequence $[13,14]$ and comparison of the consensus sequence from assembly-based approaches

\footnotetext{
* Correspondence: wuci@big.ac.cn

${ }^{1}$ Laboratory of Disease Genomics and Individualized Medicine, Beijing Institute of Genomics, Chinese Academy of Sciences, Beijing, P.R. China ${ }^{7}$ Department of Ecology and Evolution, University of Chicago, Chicago, IL, USA

Full list of author information is available at the end of the article
}

to a reference sequence [15]. While most of these methods are comprehensive, their detection ability is limited on medium-sized deletions at low sequencing coverage. Furthermore, many analyses do not require a comprehensive genomic survey, but identify certain specific markers. For example, in comparative genomic studies, a small subset of deletions is sufficient to serve as molecular markers to trace the evolution of genomes. During the past five years, several restriction enzyme based methods have been developed for this purpose, including RRL [16], RAD-seq [17], CroPS [18] and GBS [19]. Most of them aimed at identifying single nucleotide polymorphism (SNP) in restriction regions. However, few methods were developed to detect proportional deletions at the genomic level.

Chen et al. proposed a method for examining genomic structural variations based on paired-end restriction tags (ditags) [20]. However, its application was limited due to complex experimental procedures requiring laborious library cloning and single-end sequencing. This method also lacked a computational program for variant discovery. Taking the advantage of new NGS developments, we greatly simplified the library construction process by
Ciomed Central

(c) 2013 Gong et al.; licensee BioMed Central Ltd. This is an Open Access article distributed under the terms of the Creative Commons Attribution License (http://creativecommons.org/licenses/by/2.0), which permits unrestricted use, distribution, and reproduction in any medium, provided the original work is properly cited. 


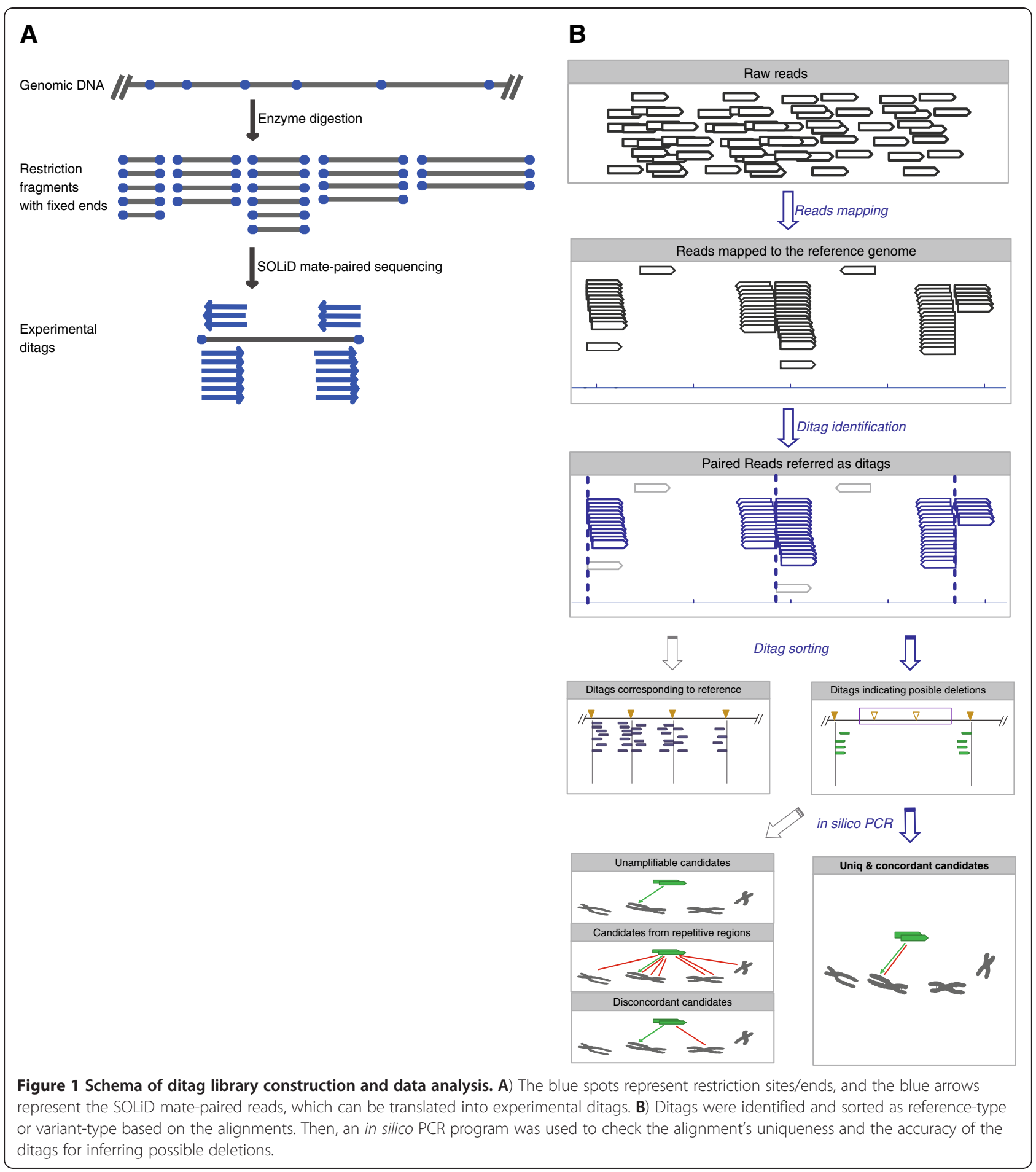

adapting this method to a mate-paired library construction system for sequencing and validation (Figure 1A). We also developed a computational program to identify genomic deletions and an experimental protocol to verify the mapped deletions. We used this system to analyze a liver cancer genome. The results demonstrated the power of the system.

\section{Results}

\section{The major improvements in our method}

Compared to the previous method [20], we made four major improvements:

1) We use paired-end sequencing instead of single-end sequencing. 
2) We adopted a mate-paired system for library construction to eliminate the cloning process.

3) We extended the sequence length beyond the original 17 bp limit.

4) We designed a specific computational program for ditag analysis and deletion detection.

Therefore, our improved method is more simple, specific and systematic.

\section{Choice of restriction enzyme determines the detection resolution}

The goal of our method is to detect medium-sized deletions, specifically those in the range of $100 \mathrm{bp}$ to $10 \mathrm{~kb}$.

The detection resolution is correlated with the cutting frequencies of the selected restriction enzymes (Figure 2). Each enzyme recognizes fixed restriction sites and produces fixed restriction tags; thus, it targets a fixed proportion of the deletions. For normal fragments, the paired tags are located at two adjacent restriction sites, whereas deletions that include restriction sites result in consecutive skipping of those sites. Skipping a single restriction site can either be attributed to a point mutation that has caused its inactivation or to an undigested site (due to its partial digestion). To increase the specificity, we only detected deletions that skipped at least two consecutive restriction sites.

We simulated the restriction digestion of 164 unique restriction sites to test their detection resolutions (Figure 2A; Additional file 1: Table S1). The sites are recognized by more than 4,000 type II restriction enzymes in REBASE (http://rebase.neb.com/cgi-bin/azlist?re2). The results show that the detection resolutions of two known deletion datasets (Methods) are strongly correlated with each enzyme's cutting frequency. Furthermore, up to $80 \%$ of the deletions can be targeted by the most frequent cutter.

TaqI, which recognizes the sequence TCGA, produces 1.5 million fragments with a median size of $1.2 \mathrm{~kb}$. Tests with this enzyme show that $10 \%$ and $28 \%$ of the medium-sized deletions can be target detected in the $Y H$ genome and the $D G V$ database, respectively (Figure $2 \mathrm{~B}$ ). An overview of the analysis pipeline is shown in Figure 1B.

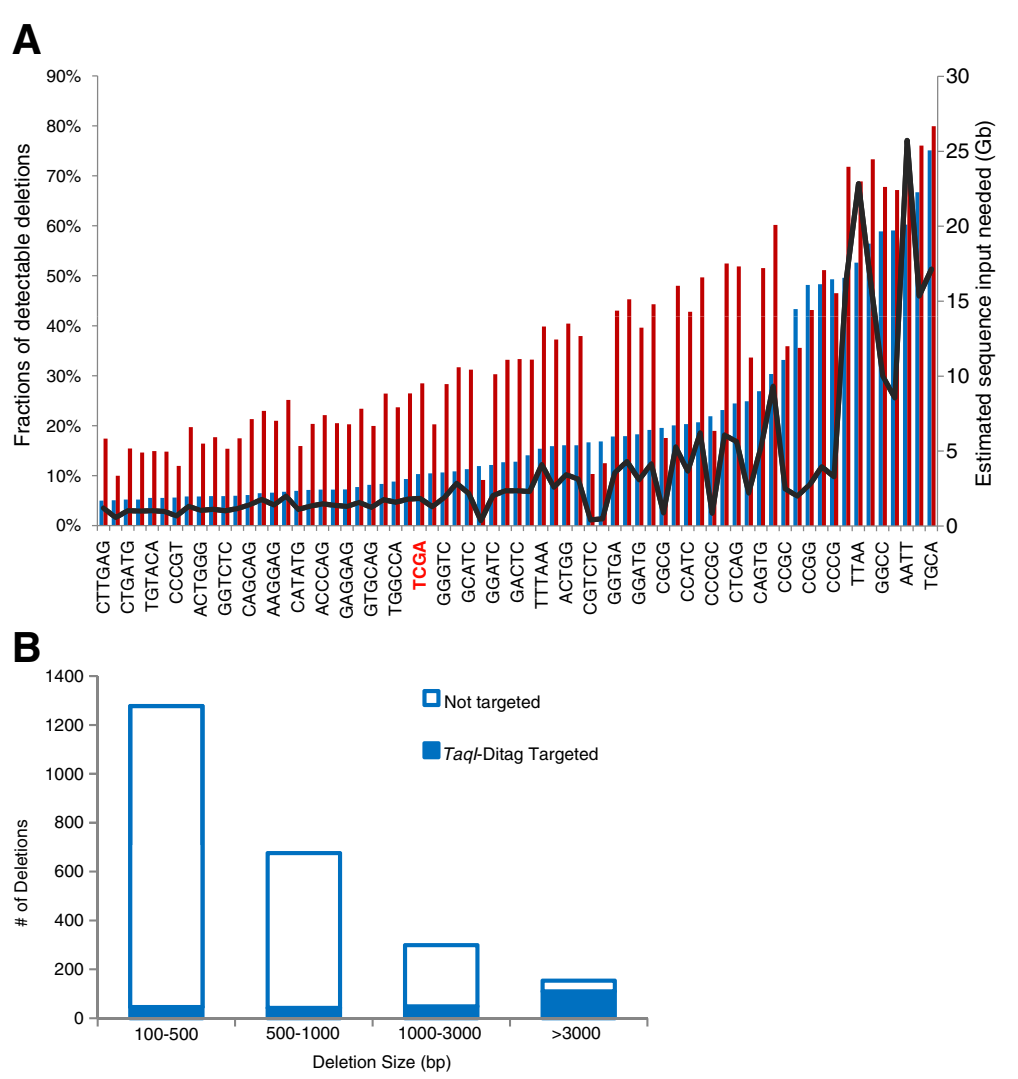

Figure $\mathbf{2}$ In silico analysis of detected deletions by restriction ditags. A) Evaluation of the detection resolution of deleted restriction sites with a wide range of cutting frequencies. The bar plot represents the fraction of detectable deletions associated with restriction sites in the $\mathrm{YH}$ genome (blue) and the DGV database (red). The dark line represents the estimated sequence input for various restriction sites, assuming that the restriction ends are covered by an average of $12 \times$ paired 50-bp reads. B) The fraction of deletions that are targeted by Taql ditags (cutting at T, (GA) across various size ranges in $Y H$ genome. 


\section{Consistency of restriction ditags from the two libraries}

We analyzed a liver cancer genome using a TaqI restriction digestion. We constructed two separate libraries, producing 9.4 million read pairs of $33 \times 2$ bases in Library 1 and 24.7 million read pairs of $48 \times 2$ bases in Library 2 (Table 1 ).

Overall, 29 million sequences (85\%) were mapped to the expected restriction sites in the hg18 reference genome sequence (ditags), while the other 15\% of the reads failed to map to the expected regions. The hg18 reference contains 1,509,487 unique ditags that are defined by the TaqI sites (Ref-Ditags). Approximately $68 \%$ of the Ref-Ditags were mapped by experimental ditags with an average depth of $17.7 \times$ (Table 1 ). The 18 million experimental ditags cover $45 \%$ of the genome.

The ditags from the two libraries were highly consistent, covering $53 \%$ and $65 \%$ of the Ref-Ditags. While the coverage percentages were not high, the proportions of their covered genomic regions are highly correlated across different chromosomes (Additional file 2: Figure S1). Furthermore, $50 \%$ of the Ref-Ditags were covered by both datasets with a $73 \%$ rate of overlap between the datasets (Additional file 2: Figure S2A). Analysis of the insert size distribution showed that the overlapping ditags tended to be small fragments (Figure 3A). Both libraries showed correlated coverage-enrichment curves for their ditags (Figure 3B), which could be attributed to the fact that smaller fragments were more likely to be circularized than larger fragments [21]. This graph also explains the presence of uncovered Ref-Ditags, which have significantly larger insert sizes than covered Ref-Ditags (Figure 3A). In effect, this feature enhances the reproducibility of the restrictionbased method by targeting fragments of a given size.

\section{Deletion identification}

The following four conditions were used to identify the candidate deletions.
1) At least two consecutive restriction sites were skipped by the ditags (Figure 4) and the two restriction sites should include at least one site that is excluded from the SNP database (dbSNP, build130). This criterion should prevent false positive results raised by random point mutations that inactivate restriction sites.

2) A candidate deletion should be supported by at least two ditags in order to eliminate artifact from the randomness during both experimental and computational process.

3) Ditags should pass the in silico PCR test (isPCR; http://genome.ucsc.edu/cgi-bin/hgPcr). This test simulates the real PCR process by searching all possible genomic alignments within an expected distance and allows multiple mismatches. We used isPCR to examine the accuracy of the ditag sequences as sense/antisense primers. Ditags remained if they produced a single electronic $\mathrm{PCR}$ product at the alignment position (Figure 1B). This step ensures the reliability of the mapping result as well as the success rate of the downstream $P C R$ validation.

4) A candidate deletion should be supported by more than $10-40 \%$ of the ditags mapped to its locus. Candidate deletions with low proportion of the ditags mapped to the locus were eliminated. These candidates could represent deletions from duplicate regions, making them difficult to validate. However, setting it too high would have a danger of losing real heterozygous deletions. In principle, the threshold mainly depends on the complexity, especially the repetitive content, of the in-analysis genome. In our analysis, we set this value to $33 \%$ to ensure a high specificity.

Using these conditions, we identified 51 and 150 deletions from the two libraries, with a total of 175 deletions

Table 1 Statistics on read coverage and the identified deletions

\begin{tabular}{llll}
\hline & Library 1 (33x2) & Library 2 (48x2) & Combined library \\
\hline Raw reads & $45.9 \mathrm{M}^{1} / 1.51 \mathrm{~Gb}^{2}$ & $148 \mathrm{M} / 7.11 \mathrm{~Gb}$ & $194 \mathrm{M} / 8.63 \mathrm{~Gb}$ \\
Mapped reads & $24.3 \mathrm{M} / 0.80 \mathrm{~Gb}$ & $68.4 \mathrm{M} / 3.28 \mathrm{~Gb}$ & $92.7 \mathrm{M} / 4.08 \mathrm{~Gb}$ \\
Read pairs & $9.41 \mathrm{M} / 0.62 \mathrm{~Gb}$ & $24.7 \mathrm{M} / 2.37 \mathrm{~Gb}$ & $34.1 \mathrm{M} / 2.99 \mathrm{~Gb}$ \\
Ditags & $7.66 \mathrm{M} / 0.51 \mathrm{~Gb}$ & $21.3 \mathrm{M} / 2.05 \mathrm{~Gb}$ & $29.0 \mathrm{M} / 2.55 \mathrm{~Gb}$ \\
Ditags mapped to Ref-Ditags & $6.73 \mathrm{M} / 0.44 \mathrm{~Gb}$ & $11.4 \mathrm{M} / 1.09 \mathrm{~Gb}$ & $18.1 \mathrm{M} / 1.53 \mathrm{~Gb}$ \\
Ref-Ditags identified (1,509,487 in all) & $794,515(53 \%)$ & $981,480(65 \%)$ & $1,024,072(68 \%)$ \\
Average ditag depth on each Ref-Ditag & 8.47 & 11.57 & 17.66 \\
Genomic regions covered (Mb) & $834(28 \%)$ & $1,274(42 \%)$ & $1,336(45 \%)$ \\
Median fragment length (bp) & 880 & 1,056 & 1,056 \\
Deletions identified & $51(29 \%)$ & $150(86 \%)$ & 175 \\
Average ditag depth on each deletion & 5.37 & 4.53 & 5.70
\end{tabular}

${ }^{1} \mathrm{M}$ : counts of the reads, pairs or ditags in millions.

${ }^{2} \mathrm{~Gb}$ : Gigabases.

${ }^{3}$ Genomic regions identified in the insert regions of the experimental ditags that correspond to the reference genome. 


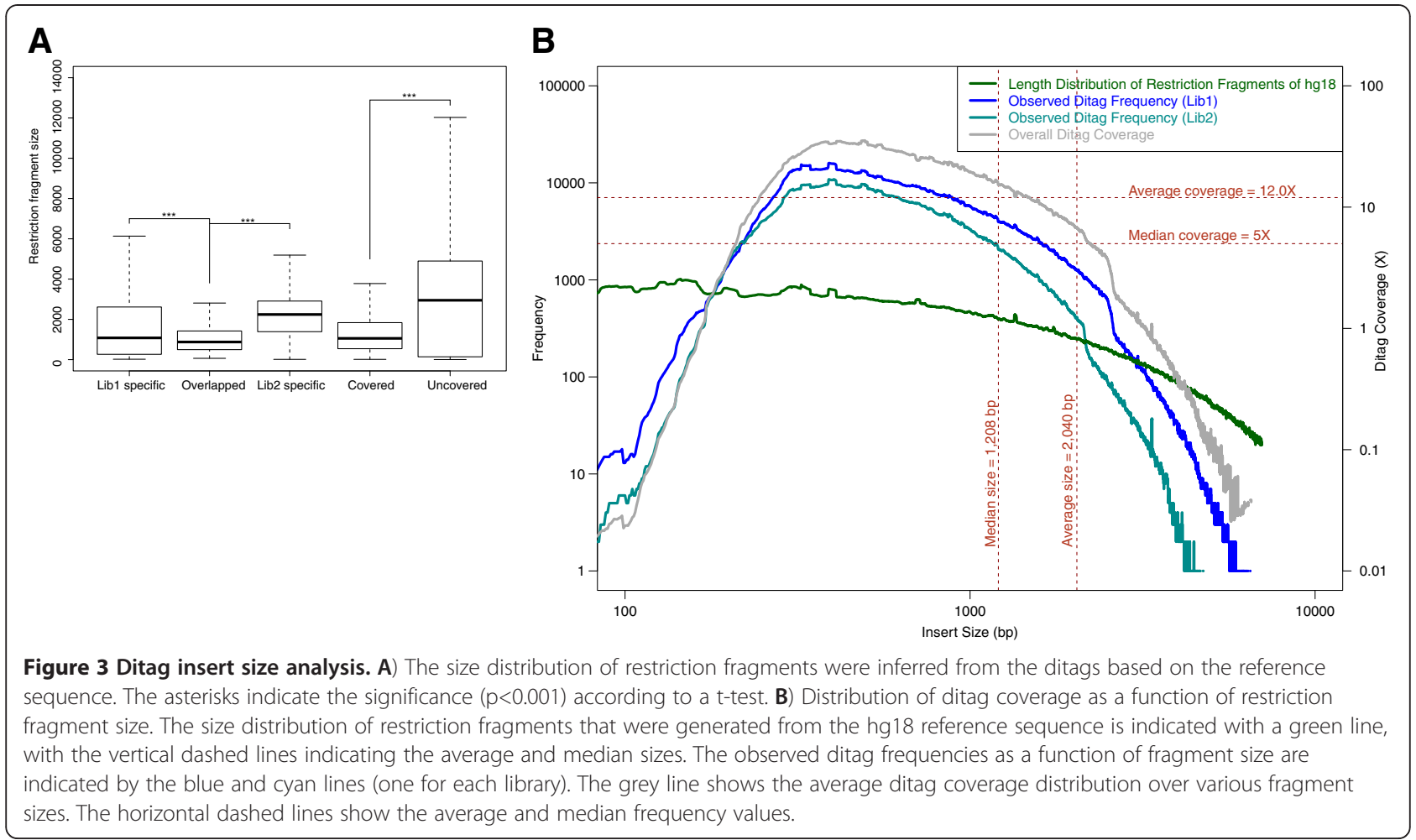

in the combined dataset (Additional file 3: Table S2). Approximately $76 \%$ of the deletions identified by the lower-coverage library were also identified by the highercoverage library (Additional file 2: Figure S2B).

\section{Validation of the candidate deletions}

We validated the candidates by PCR (Figure 4; Additional file 2: Figure S3). Of the 19 candidates randomly selected for validation, 18 were validated as real homozygous or heterozygous deletions (Table 2). The false positive one was due to the reason that one of the restriction sites was inactivated by a point mutation while the other site was also a SNP site.

Of the 18 validated deletions, 13 deletions overlapped with existing data in the Database of Genomic Variants [5]. The data indicate the involvement of the genes LRP5, ADIPOR2 and RPH3AL (Table 2), which have a reported role in developmental disorders and tumorigenesis [22-24].

According to our validation rate, the total number of actual deletions that were identified by TaqI restriction fragments was estimated to be $175 \times 18 / 19=166$.

Our simulation showed that TaqI ditag sequencing may detect up to $10 \%$ of the deletions across the entire

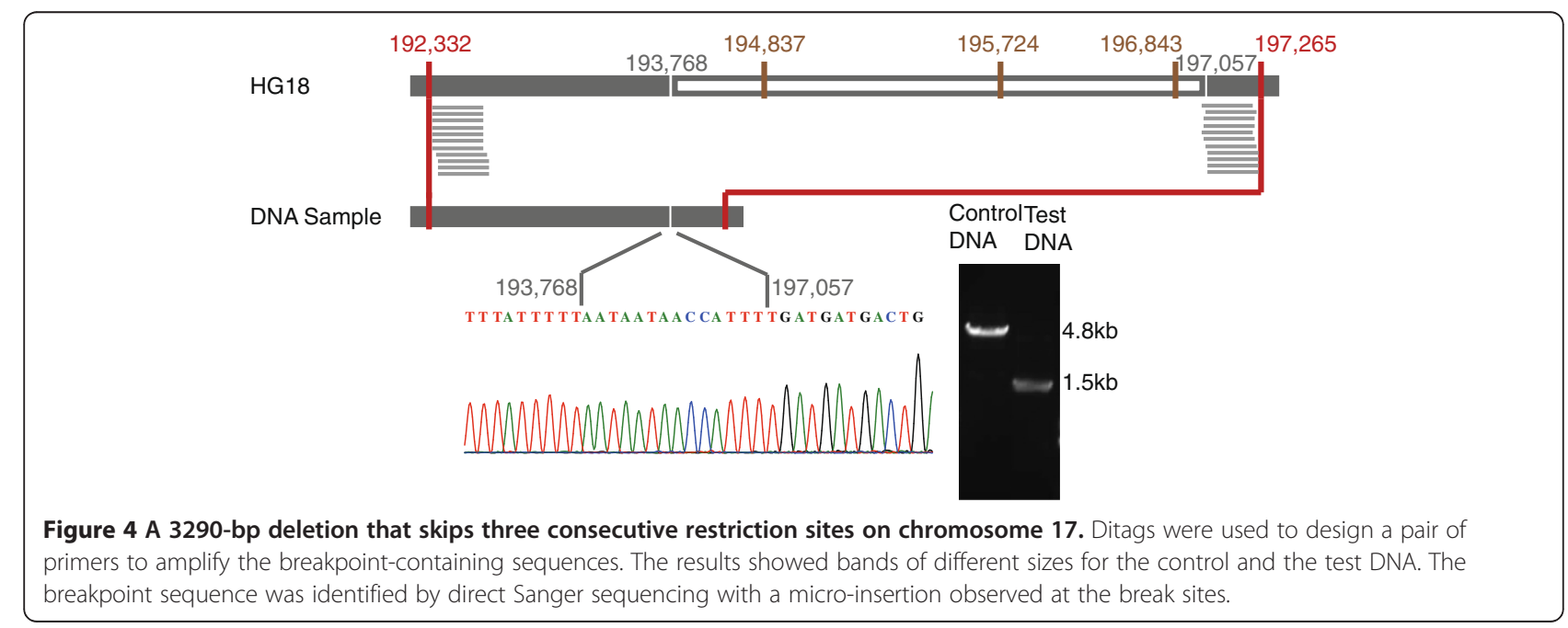


Table 2 Validation of the candidate deletions

\begin{tabular}{|c|c|c|c|c|c|}
\hline $\operatorname{ditagID}^{1}$ & Deletion breakpoints & DelSize (bp) & Notes & Gene involved & DGV overlap ${ }^{2}$ \\
\hline 1_67822_2/1_67825_1 & chr1:147489387-147489688 & 302 & Heterozygote & Intergenic & Novel \\
\hline 1_110491_2/1_110494_1 & chr1:232385269-232386371 & 1103 & Homozygote & SLC35F3 & Variation_109897 \\
\hline 1_110644_2/1_110647_1 & chr1:232653827-232654136 & 310 & Heterozygote & TARBP1 & Novel \\
\hline 5_79470_2/5_79473_1 & chr5:172632846-172633171 & 326 & Homozygote & Intergenic & Variation_46815 \\
\hline 7_50712_2/7_50719_1 & chr7:96313845-96319938 & 6094 & Homozygote & Intergenic & Variation_23855 \\
\hline 7_83664_2/7_83673_1 & chr7:158193509-158197522 & 4014 & Heterozygote & Intergenic & Variation_43560 \\
\hline 8_33206_2/8_33210_1 & chr8:64316994-64318575 & 1582 & Homozygote & Intergenic & Novel \\
\hline 9_54312_2/9_54319_1 & chr9:129221441-129225898 & 4458 & Homozygote & ZNF79 & Variation_106098 \\
\hline 11_33186_2/11_33189_1 & chr11:67939676-67939985 & 310 & Homozygote & LRP5 & Novel \\
\hline 12_1219_2/12_1222_1 & chr12:1734274-1734588 & 315 & Heterozygote & ADIPOR2 & Variation_11592 \\
\hline 12_63591_2/12_63594_1 & chr12:123274954-123275072 & 118 & Heterozygote & Intergenic & Variation_11646 \\
\hline 14_29251_2/14_29254_1 & Not Validated & 0 & $\mathrm{DPV}^{3}$ & - & - \\
\hline 17_141_2/17_145_1 & chr17:193768-197057 & 3290 & Homozygote & RPH3AL & Variation_25792 \\
\hline 17_6977_2/17_6981_1 & chr17:8187338-8188694 & 1357 & Homozygote & ODF4 & Variation_43957 \\
\hline 17_47495_2/17_47501_1 & chr17:71873831-71876905 & 3075 & Heterozygote & Intergenic & Variation_77728 \\
\hline 19_22244_2/19_22248_1 & chr19:34642031-34648013 & 5983 & Homozygote & Intergenic & Variation_43984 \\
\hline 22_10635_2/22_10639_1 & chr22:30106417-30110625 & 4209 & Homozygote & Intergenic & Variation_43568 \\
\hline X_7293_2/X_7300_1 & chrX:11635278-11641324 & 6047 & Homozygote & Intergenic & Variation_22612 \\
\hline X_21618_2/X_21622_1 & chrX:39977151-39979777 & 2627 & Homozygote & Intergenic & Novel \\
\hline
\end{tabular}

${ }^{1}$ An ID of a ditag from which the local structural information is inferred (Method).

${ }^{2}$ Deletions that overlap with structural changes in the Database of Genomic Variants [5].

${ }^{3}$ Double point variants inactivating both restriction sites.

genome (Figure 2B; Additional file 1: Table S1). Approximately $45 \%$ of the genome has been examined with the experimental ditags (Table 1). We can calculate that the lower bound on the total number of 0.1-10 kb deletions is $166 / 10 \% / 45 \%=3,684$.

\section{Discussion}

Next-generation sequencing has been a powerful tool for deletion identification [25]. A variety of computational algorithms have been developed to use NGS sequence data to search for deletions. Notably, these methods attempt to collect comprehensive details about genomic structural variants. For example, a recent study surveyed structural changes (ranging in size from single base pairs to several $\mathrm{Mbp}$ ) in two personal genomes using the de novo assembly of short reads [15]. However, comprehensive methods require high sequence coverage $(>30 \times$ genome size), which drives up costs, requires a large amount of data storage space, necessitates long analysis time and creates heavy computational demands. In select studies such as evolutionary genomics, it is not necessary to achieve comprehensiveness; instead, a limited amount of information is sufficient. In recent years, several restriction-based NGS methods have been developed to sequence partial genomes [16-19]. Most of these methods aim for SNP discovery, not the detection of structural changes. We modified the method of Chen et al. [20] by simplifying its experimental procedures and developing a computational program. In this study, we showed that sequencing both ends of the restriction fragments generated by a medium-frequency enzyme can be an accurate method for identifying medium-sized deletions, even with sequence coverage as low as one-fold genome size.

The deletion resolution can be controlled by selecting restriction enzymes with different cutting frequencies depending on the research objectives. The selected restriction enzyme determines what target regions will be sequenced, as well as the length distribution of the restriction fragments (Figure 3B). In this study, very low sequencing coverage ( $3 \mathrm{~Gb}$ or $1.0 \times$ human genome size) could be concentrated within the tag regions to reach a sufficient depth for deletion identification. The high rate of overlap between the two separate datasets used in our study, both of which had low genomic coverage, demonstrates the reproducibility of this method (Additional file 2: Figure S2).

The number of detected deletions can also be adjusted by the coverage. Library 1 contained $0.21 \times$ paired reads and detected 51 deletions, whereas Library 2 had $0.79 \times$ read coverage and detected 150 deletions (Table 1). Importantly, most of the deletions found with Library 1 were also identified with Library 2 (Additional file 2: Figure S2B). 
In addition to high flexibility and efficiency, this method also displayed high accuracy. The use of in silico PCR significantly increased the specificity of the detected deletions by eliminating the noisy sequences that were produced by experimental errors, such as randomly broken fragment ends, star activity of the restriction enzyme, sequencing errors and false mapping.

The population of target deletions can be fixed once the restriction enzyme is determined, and the size of the deletion population can be adjusted by selecting different enzymes and coverage according specific needs (Figure 1A). The flexible choice of the fixed target enabled comparative genomic studies on a subset of deletions across different samples because these deletions were randomly distributed across the genomes (Additional file 3: Table S2) and could be accessed repetitively without heavy sequencing input. Thus, our method is applicable to a variety of fields, including:

1) Detecting the deletions across multiple genomes, especially for the species with large, difficult-tosequence genomes in population or comparative genomic studies. For example, a recent survey of the structural variants in an individual gorilla genome required a $60 \mathrm{~Gb}$ sequence input [3]. At this scale, our method can examine the genetic diversity of deletions in a population of 5-20 gorillas. Although the Genome STRiP can also examine deletions in multiple large genomes, as it did with 1000 Genome data [26], it cannot deal with single genome data nor identify singletons from pooled data as we did in this study.

2) Detecting the deletions in paired samples. For example, rapid identification of residual alleles of cancer cells which usually exist in trace amount in circulating DNA [27]. By sequencing the ditags of original tumor DNA and normal DNA in the same individual, several somatically-acquired, tumor specific deletions could be identified. PCR primers could be designed based on these deletions to amplify the tumor DNA specifically.

3) Massive validation of deletions found by other comprehensive methods or massive genotyping of known deletions.

\section{Conclusions}

We developed a simplified experimental protocol and computational pipeline to detect genomic deletions at low genomic coverage. The library construction procedure can be adapted to other NGS platforms. The method is cost-effective, flexible and accurate. Our method may be potentially useful for the identification of representative markers.

\section{Methods}

\section{Materials}

Tumor tissue was surgically collected from a 52-year-old man diagnosed with hepatocellular carcinoma (HCC) at the Cancer Center, Sun Yat-sen University (Guangzhou, China). The primary tumor was $10 \times 8 \times 8 \mathrm{~cm}$, grade II to III, and showed invasive cirrhosis. Total genomic DNA was isolated using a standard protocol with proteinase digestion, phenol-chloroform extraction and ethanol precipitation. The study was approved by the Institutional Review Board, and informed consent was signed by the patient.

\section{Simulating the detection resolution of various enzymes}

Two test datasets were used. Both included deletions that were characterized in previous studies. The first set of deletions was from an Asian genome ( $Y H$ genome) (http: // yh.genomics.org.cn/do.downServlet?file=data/sv/ YHsv.gff), which included a total of 2,403 median-sized deletions (0.1-10 kb) across the genome [28]. The second dataset was from the Database of Genomic Variants (http://projects.tcag.ca/variation/downloads/) in the files variation.hg18.v10.nov.2010.txt and indel.hg18. v10.nov.2010.txt, which record 66,220 median-sized deletions in multiple individual genomes. A Perl script was used to conduct the simulation (Additional file 4). The human reference sequence (hg18) was searched for the restriction sites of the given enzymes. Deletions covering two or more sites were classified as detectable by the enzymes. The detection resolution was defined as the proportion of detectable deletions in each test dataset (Figure 2).

\section{Mate-paired library construction}

Additional file 2: Figure S4 illustrate the overall steps of the library construction. Ten micrograms of genomic DNA were mixed with $30 \mu \mathrm{L}$ of $10 \times$ Buffer E (Promega), $3 \mu \mathrm{L}$ of acetylated BSA $(10 \mu \mathrm{g} / \mu \mathrm{L}$, Promega), $7.5 \mu \mathrm{L}$ of TaqI (10 U/ $\mu \mathrm{L}$, Promega), and nuclease-free water to reach a total volume of $300 \mu \mathrm{L}$. The initial amount of genomic DNA was determined by the average insert size of the restriction fragments, which should be consistent with the amount requirement of a standard mate-paired library. The mixture was incubated at $65^{\circ} \mathrm{C}$ for four hours.

Restriction fragments that were $200-6000$ bp in length were selected on a $1 \%$ agarose gel and purified using the Gel Purification Kit (Qiagen). Purified restriction fragments were attached with sticky CAP adapters that were modified from standard SOLiD CAP adapters [5'- CGC TGC TGT AC -3' (positive strand); 5' - ACA GCA G - $3^{\prime}$ (negative strand); $100 \mu \mathrm{M}$ ]. Then, $8.3 \mu \mathrm{L}$ of sticky CAP adaptors, $5.3 \mu \mathrm{g}$ of DNA restriction fragments, $300 \mu \mathrm{L}$ of $2 \times$ quick ligase buffer, $15 \mu \mathrm{L}$ of quick ligase $(N E B)$ and nuclease-free water were mixed and incubated at room 
temperature for 10 minutes. The ligation products were purified using the Gel Purification Kit (Qiagen). Adapter-ligated restriction fragments were then applied the standard mate-paired library construction procedure. The sequencing reaction was conducted following the manufacturer's protocol. The $2 \times 33$ reads were collected on SOLiD 2, and the $2 \times 48$ reads were collected on SOLiD 3.

\section{Sequence mapping}

SOLiD color space reads were mapped to the human reference genome (hg18) using the BWA program (v0.5.9) with the default options for color space mapping [29]. Only pairs in which both sequences mapped to the reference were used for downstream analysis.

The SOLiD mated-paired library construction process will result in one read that is sequenced from the exact end of the fragment and another read that is sequenced a distance away from the fragment end. Thus, one member of the read pair should map to the exact position of the restriction digestion, while the other member should map approximately 100-200 bp away from this position as a result of the 'nick-translation' procedure (Additional file 2: Figure S4). A nominal distribution was inferred from the mapping results, reflecting the nick-translated distances (Additional file 2: Figure S5).

\section{Translating sequence reads into clusters of ditags}

In our algorithm, we created an ID system to separate the normal and variant ditags. All of the reads that mapped to expected restriction sites had a reference to the restriction site's ID. An ID includes chromosomal information, the serial number of the corresponding restriction site and the relative position of both tags. For example, ID \#3-25-2 and ID \#3-26-1 represent the downstream region of the $25^{\text {th }}$ TCGA-site and the upstream region of the $26^{\text {th }}$ TCGA-site, respectively, along chromosome 3 . Both regions supposedly correspond to the same restriction fragment, as defined by their hg18 reference. Genomic structure was inferred by reading the information from both ditag IDs. For example, a ditag formed by $\# A-N-2$ and $\# A-(N+1)-1$ represents a pair corresponding to the reference structure, while a ditag formed by $\# A-N-2$ and $\# A-(N+4)-1$ represents a pair that skips three consecutive restriction sites, indicating a possible deletion on chromosome $A$. See Additional file 4 for the original scripts.

\section{Validation by PCR amplification and clone sequencing}

PCR primers were designed based on the ditags using Primer Premier 5 software (Additional file 5: Table S3). PCR reactions included $\sim 5 \mathrm{ng}$ of genomic DNA, $2 \mu \mathrm{L}$ of forward primer $(10 \mu \mathrm{M}), 2 \mu \mathrm{L}$ of reverse primer $(10 \mu \mathrm{M}), 5 \mu \mathrm{L}$ of $10 \times$ LA Taq Buffer (Takara), $8 \mu \mathrm{L}$ of
dNTP $(2.5 \mathrm{mM}), 2 \mu \mathrm{L}$ of LA Taq polymerase (Takara) and nuclease-free water to reach a volume of $50 \mu \mathrm{L}$. Touch-down PCR was used to amplify the products. The conditions included a 5 -min denaturing at $95^{\circ} \mathrm{C}$ followed by $4 \times 5$ cycles of $30 \mathrm{sec}$ at $95^{\circ} \mathrm{C}, 40 \mathrm{sec}$ at $64^{\circ} \mathrm{C}$, $62^{\circ} \mathrm{C}, 60^{\circ} \mathrm{C}$ and $58^{\circ} \mathrm{C}$ for each group of 5 cycles, and $2-5 \mathrm{~min}$ at $72^{\circ} \mathrm{C}$ for elongation, and then 20 cycles of $30 \mathrm{sec}$ at $95^{\circ} \mathrm{C}, 40 \mathrm{sec}$ at $56^{\circ} \mathrm{C}, 5 \mathrm{~min}$ at $72^{\circ} \mathrm{C}$ for elongation, and $10 \mathrm{~min}$ at $72^{\circ} \mathrm{C}$. The elongation time was dependent on the expected product size and is based on the reference genome, which was calculated as [\# $\mathrm{Kb}]$ min. The amplified products were checked on $1 \%$ agarose gels. The selected PCR products were purified from the gel, cloned to the pGEM-T Vector (Promega) and used for sequencing via a big-dye reagent.

\section{Data availability}

The sequences from this paper have been submitted to the NCBI Short Reads Archive (http://www.ncbi. nlm.nih.gov/Traces/sra/sra.cgi) under accession number SRA058045.

\section{Additional files}

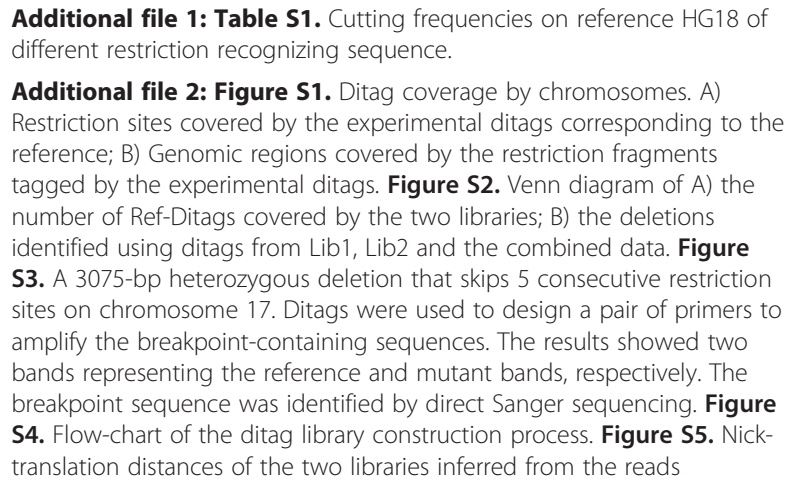

Additional file 2: Figure S1. Ditag coverage by chromosomes. A) Restriction sites covered by the experimental ditags corresponding to the reference; B) Genomic regions covered by the restriction fragments tagged by the experimental ditags. Figure S2. Venn diagram of A) the number of Ref-Ditags covered by the two libraries; B) the deletions identified using ditags from Lib1, Lib2 and the combined data. Figure S3. A 3075-bp heterozygous deletion that skips 5 consecutive restriction sites on chromosome 17. Ditags were used to design a pair of primers to amplify the breakpoint-containing sequences. The results showed two bands representing the reference and mutant bands, respectively. The breakpoint sequence was identified by direct Sanger sequencing. Figure S4. Flow-chart of the ditag library construction process. Figure S5. Nicktranslation distances of the two libraries inferred from the reads alignment.

Additional file 3: Table S2. A list of the identified medium-sized deletions.

Addtional file 4: A package of scripts and related programs conducting the analysis.

Additional file 5: Table S3. Primers used for validation.

Competing interests

The authors declare that they have no competing interests.

\section{Authors' contributions}

QG, YT, XL, SMZ, SMW and CIW designed the studies. SMZ and YY provided the tumor sample and extracted the genomic DNA. QG, YT, HL and JY constructed the library. QG, JRY, WL and JC analyzed the data. QG developed the pipeline and performed the experimental validation. QG, JC, JR, SMW and CIW drafted the manuscript. XL and CIW coordinated and supervised the study. All authors read and approved the final manuscript.

\section{Acknowledgements}

This study was supported by the National Natural Science Foundation of China Grant 91131903, 31171265 and National Basic Research Program of China Grant 2012CB316505. 


\section{Author details}

${ }^{1}$ Laboratory of Disease Genomics and Individualized Medicine, Beijing Institute of Genomics, Chinese Academy of Sciences, Beijing, P.R. China. ${ }^{2}$ University of Chinese Academy of Sciences, Beijing, P.R. China. ${ }^{3}$ Key Laboratory of Gene Engineering of the Ministry of Education, State Key Laboratory of Biocontrol, School of Life Science, Sun Yat-sen University, Guangzhou, P.R. China. ${ }^{4}$ Department of Hepatobiliary Oncology, Cancer Center, Sun Yat-sen University, Guangzhou, P.R. China. ${ }^{5}$ Chinese Academy of Sciences Key Laboratory of Genome Sciences and Information, Beijing Institute of Genomics, Chinese Academy of Sciences, Beijing, P.R. China. ${ }^{6}$ Department of Genetics, Cell Biology \& Anatomy, College of Medicine, University of Nebraska Medical Center, Nebraska, USA. ${ }^{7}$ Department of Ecology and Evolution, University of Chicago, Chicago, IL, USA.

Received: 4 September 2012 Accepted: 18 January 2013 Published: 24 January 2013

\section{References}

1. Emerson JJ, Cardoso-Moreira M, Borevitz JO, Long M: Natural selection shapes genome-wide patterns of copy-number polymorphism in Drosophila melanogaster. Science 2008, 320:1629-1631.

2. Feuk L, Carson AR, Scherer SW: Structural variation in the human genome. Nature 2006, 7:85-97.

3. Ventura M, Catacchio CR, Alkan C, Marques-Bonet T, Sajjadian S, Graves TA, Hormozdiari F, Navarro A, Malig M, Baker C, Lee C, Turner EH, Chen L, Kidd JM, Archidiacono N, Shendure J, Wilson RK, Eichler EE: Gorilla genome structural variation reveals evolutionary parallelisms with chimpanzee. Genome Res 2011, 21:1640-1649.

4. Hurles ME, Dermitzakis ET, Tyler-Smith C: The functional impact of structural variation in humans. Trends Genet 2008, 24:238-245.

5. lafrate AJ, Feuk L, Rivera MN, Listewnik ML, Donahoe PK, Qi Y, Scherer SW, Lee C: Detection of large-scale variation in the human genome. Nat Genet 2004, 36:949-951.

6. Sebat J, Lakshmi B, Troge J, Alexander J, Young J, Lundin P, Månér S, Massa H, Walker M, Chi M, Navin N, Lucito R, Healy J, Hicks J, Ye K, Reiner A, Gilliam TC, Trask B, Patterson N, Zetterberg A, Wigler M: Large-scale copy number polymorphism in the human genome. Science 2004, 305:525-528.

7. Itsara A, Cooper GM, Baker C, Girirajan S, Li J, Absher D, Krauss RM, Myers RM, Ridker PM, Chasman DI, Mefford H, Ying P, Nickerson DA, Eichler EE: Population analysis of large copy number variants and hotspots of human genetic disease. Am J Hum Genet 2009, 84:148-161.

8. Campbell PJ, Stephens PJ, Pleasance ED, O'Meara S, Li H, Santarius T, Stebbings LA, Leroy C, Edkins S, Hardy C, Teague JW, Menzies A, Goodhead I, Turner DJ, Clee CM, Quail MA, Cox A, Brown C, Durbin R, Hurles ME, Edwards PAW, Bignell GR, Stratton MR, Futreal PA: Identification of somatically acquired rearrangements in cancer using genome-wide massively parallel paired-end sequencing. Nat Genet 2008, 40:722-729.

9. Chiang DY, Getz G, Jaffe DB, Kelly MJTO, Zhao X, Carter SL, Russ C, Nusbaum C, Meyerson M, Lander E: High-resolution mapping of copy-number alterations with massively parallel sequencing. Nature 2009 6:99-103.

10. Tuzun E, Sharp AJ, Bailey JA, Kaul R, Morrison VA, Pertz LM, Haugen E, Hayden $\mathrm{H}$, Albertson D, Pinkel D, Olson MV, Eichler EE: Fine-scale structural variation of the human genome. Nat Genet 2005, 37:727-732.

11. Kidd JM, Cooper GM, Donahue WF, Hayden HS, Sampas N, Graves T, Hansen N, Teague B, Alkan C, Antonacci F, Haugen E, Zerr T, Yamada NA, Tsang P, Newman TL, Tüzün E, Cheng Z, Ebling HM, Tusneem N, David R, Gillett W, Phelps KA, Weaver M, Saranga D, Brand A, Tao W, Gustafson E, McKernan K, Chen L, Malig M, Smith JD, Korn JM, McCarroll SA, Altshuler DA, Peiffer DA, Dorschner M, Stamatoyannopoulos J, Schwartz D, Nickerson DA, Mullikin JC, Wilson RK, Bruhn L, Olson MV, Kaul R, Smith DR, Eichler EE: Mapping and sequencing of structural variation from eight human genomes. Nature 2008, 453:56-64

12. Korbel JO, Urban AE, Affourtit JP, Godwin B, Grubert F, Simons JF, Kim PM, Palejev D, Carriero NJ, Du L, Taillon BE, Chen Z, Tanzer A, CE Sa, Chi J, Yang F, Carter NP, Hurles ME, Weissman SM, Harkins TT, Gerstein MB, Egholm M, Snyder M: Paired-end mapping reveals extensive structural variation in the human genome. Science 2007, 318:420-426.

13. Ye K, Schulz MH, Long Q, Apweiler R, Ning Z: Pindel: a pattern growth approach to detect break points of large deletions and medium sized insertions from paired-end short reads. Bioinformatics 2009, 25:2865-2871.
14. Zhang ZD, Du J, Lam H, Abyzov A, Urban AE, Snyder M, Gerstein M: Identification of genomic indels and structural variations using split reads. BMC Genomics 2011, 12:375.

15. Li Y, Zheng H, Luo R, Wu H, Zhu H, Li R, Cao H, Wu B, Huang S, Shao H, Ma H, Zhang F, Feng S, Zhang W, Du H, Tian G, Li J, Zhang X, Li S, Bolund L, Kristiansen K, Smith AJD, Blakemore AIF, Coin LJM, Yang H, Wang JJ, de Smith AJ: Structural variation in two human genomes mapped at single-nucleotide resolution by whole genome de novo assembly. Nat Biotechnol 2011, 29:725-732.

16. Van Tassell CP, Smith TPL, Matukumalli LK, Taylor JF, Schnabel RD, Lawley CT, Haudenschild CD, Moore SS, Warren WC, Sonstegard TS, Van Tassell CP: SNP discovery and allele frequency estimation by deep sequencing of reduced representation libraries. Nat Methods 2008 5:247-252.

17. Baird NA, Etter PD, Atwood TS, Currey MC, Shiver AL, Lewis ZA, Selker EU, Cresko WA, Johnson EA: Rapid SNP discovery and genetic mapping using sequenced RAD markers. PLoS One 2008, 3:e3376.

18. Van Orsouw NJ, Hogers RCJ, Janssen A, Yalcin F, Snoeijers S, Verstege E, Schneiders H, Van der Poel H, Van Oeveren J, Verstegen H, Van Eijk MJT: Complexity reduction of polymorphic sequences (CRoPS): a novel approach for large-scale polymorphism discovery in complex genomes. PLoS One 2007, 2:e1172.

19. Elshire RJ, Glaubitz JC, Sun Q, Poland JA, Kawamoto K, Buckler ES, Mitchell SE: A robust, simple genotyping-by-sequencing (GBS) approach for high diversity species. PLOS One 2011, 6:e19379.

20. Chen J, Kim YC, Jung Y-C, Xuan Z, Dworkin G, Zhang Y, Zhang MQ, Wang SM: Scanning the human genome at kilobase resolution. Genome Res 2008, 18:751-762.

21. Collins FS, Weissman SM: Directional cloning of DNA fragments at a large distance from an initial probe: a circularization method. Proc Natl Acad Sci USA 1984, 81:6812-6816.

22. Yadav VK, Ryu J-H, Suda N, Tanaka KF, Gingrich JA, Schütz G, Glorieux FH, Chiang CY, Zajac JD, Insogna KL, Mann JJ, Hen R, Ducy P, Karsenty G: Lrp5 controls bone formation by inhibiting serotonin synthesis in the duodenum. Cell 2008, 135:825-837.

23. Liu Y, Michael MD, Kash S, Bensch WR, Monia BP, Murray SF, Otto KA, Syed SK, Bhanot S, Sloop KW, Sullivan JM, Reifel-Miller A: Deficiency of adiponectin receptor 2 reduces diet-induced insulin resistance but promotes type 2 diabetes. Endocrinology 2007, 148:683-692.

24. Schiff M, Delahaye A, Andrieux J, Sanlaville D, Vincent-Delorme C, Aboura A, Benzacken B, Bouquillon S, Elmaleh-Berges M, Labalme A, Passemard S, Perrin L, Manouvrier-Hanu S, Edery P, Verloes A, Drunat S: Further delineation of the 17p13.3 microdeletion involving YWHAE but distal to PAFAH1B1: four additional patients. Eur J Med Genet 2010, 53:303-308.

25. Alkan C, Coe BP, Eichler EE: Genome structural variation discovery and genotyping. Nat Rev Genet 2011, 12:363-376.

26. Handsaker RE, Korn JM, Nemesh J, McCarroll SA: Discovery and genotyping of genome structural polymorphism by sequencing on a population scale. Nat Genet 2011, 43:269-276.

27. Leary RJ, Kinde I, Diehl F, Schmidt K, Clouser C, Duncan C, Antipova A, Lee C, McKernan K, De La Vega FM, Kinzler KW, Vogelstein B, Diaz LA Jr, Velculescu VE: Development of personalized tumor biomarkers using massively parallel sequencing. Sci Transl Med 2010, 2:20ra14.

28. Wang J, Wang W, Li R, Li Y, Tian G, Goodman L, Fan W, Zhang J, Li J, Zhang J, Guo Y, Feng B, Li H, Lu Y, Fang X, Liang H, Du Z, Li D, Zhao Y, Hu Y, Yang Z, Zheng H, Hellmann I, Inouye M, Pool J, Yi X, Zhao J, Duan J, Zhou Y, Qin J, Ma L, Li G, Yang Z, Zhang G, Yang B, Yu C, Liang F, Li W, Li S, Li D, Ni P, Ruan J, Li Q, Zhu H, Liu D, Lu Z, Li N, Guo G, Zhang J, Ye J, Fang L, Hao Q, Chen Q, Liang Y, Su Y, San A, Ping C, Yang S, Chen F, Li L, Zhou K, Zheng H, Ren Y, Yang L, Gao Y, Yang G, Li Z, Feng X, Kristiansen K, Wong GK-S, Nielsen R, Durbin R, Bolund L, Zhang X, Li S, Yang H, Wang J: The diploid genome sequence of an Asian individual. Nature 2008, 456:60-65.

29. Li H, Durbin R: Fast and accurate short read alignment with BurrowsWheeler transform. Bioinformatics 2009, 25:1754-1760.

doi:10.1186/1471-2164-14-51

Cite this article as: Gong et al.: Identification of medium-sized genomic deletions with low coverage, mate-paired restricted tags. BMC Genomics $201314: 51$ 